\title{
UTILIZAÇÃO DO RESÍDUO INDUSTRIAL FERKAL NA PRODUÇÃO DE MUDAS DE Mimosa caesalpiniaefolia, EM ESTÉRIL DE EXTRAÇÃO DE ARGILA, INOCULADAS COM FUNGOS MICORRÍZICOS ARBUSCULARES E RIZÓBIO(1)
}

\author{
A. Z. PRALON ${ }^{(2)} \&$ M. A. MARTINS(3)
}

\begin{abstract}
RESUMO
Realizou-se um experimento em casa de vegetação com o objetivo de avaliar os efeitos da inoculação com fungos micorrízicos arbusculares (FMAs) e, ou, rizóbio, associados à adição de resíduo da fabricação de ácido láctico (Ferkal), na produção de mudas de Mi mosa caesal pi ni aefolia (sabiá) em estéril de extração de argila. Foram utilizados vasos plásticos de $6 \mathrm{~L}$ que continham estéril de extração de argila, adicionado do resíduo Ferkal (nas concentrações de 0, 50, 100 e $200 \mathrm{~g} \mathrm{dm}^{-3}$ ). Foram empregados seis tratamentos microbiológicos (F MAs nativos; F MA Glomus clarum; rizóbio; F MAs nativos + rizóbio; FMA G. clarum + rizóbio, e controle não inoculado). Após 103 dias, as mudas foram coletadas, e analisados - peso da matéria seca dos nódulos, a taxa de colonização micorrízica, o peso da matéria seca e os teores de $\mathbf{N}$ e $\mathbf{P}$ na parte aérea das mudas. Os resultados demonstraram que adição de Ferkal no tratamento-controle (sem inoculação) aumentou significativamente o teor de P. Entretanto, as mel hores respostas foram obtidas nas mudas inoculadas com F MAs e, ou, rizóbio, que apresentaram, em relação ao controle, aumentos significativos no peso da matéria seca e nos teores de $\mathbf{N}$ e $\mathbf{P}$ da parte aérea das mudas, em quase todos os tratamentos inoculados. Os FMAs nativos foram mais eficientes que o FMA G. clarum em promover o crescimento de M. caesalpiniaefolia.
\end{abstract}

Termos de indexação: micorrizas, áreas degradadas, fósforo, nitrogênio.

(1) Parte da Tese de Mestrado do primeiro autor, apresentada à Universidade Estadual do Norte Fluminense - UE NF. Recebido para publicação em julho de 1999 e aprovado em agosto de 2000.

(2) Mestre em Produção Vegetal pela Universidade Estadual do Norte Fluminense - UENF. CEP 28015-620 Campos dos Goytacazes (RJ).

(3) Professor Associado, UENF/CCTA. Email: marco@uenf.br. 


\begin{abstract}
SUMMARY: USE OF THE INDUSTRIAL WASTE FERKAL IN THE PRODUCTION OF Mimosa caesalpiniaefolia SEEDLINGS, IN DEGRADED SOIL FROM CLAY EXTRACTION AREAS, INOCULATED WITH ARBUSCULAR MYCORRHIZAL FUNGI AND RHIZOBIUM
\end{abstract}

\begin{abstract}
An experiment was carried out under greenhouse conditions to evaluate the effects of arbuscular mycorrhizal fungi (AMF) and, or rhizobium inoculation, with the addition of industrial waste from lactic acid production (Ferkal), on the growth of Mimosa caesal piniaefolia. Plastic pots of $6 \mathrm{~L}$ of capacity were used, filled with degraded soil from clay extraction areas with four levels of the Ferkal residue (0, 50, 100 and $\left.200 \mathrm{~g} \mathrm{dm}^{-3}\right)$. Six microbiological treatments were conducted: indigenous AMF; AMF Glomus clarum; rhizobium; indigenous AMF + Rhizobium; AMF G. clarum + Rhizobium; and, non-inoculated control. Theresul ts wereevaluated 103 days after planting by measuring AMF col onization; dry matter of nodules in theroots; weight of dry matter and $\mathrm{N}$ and $\mathrm{P}$ contents in theshoots. Theresults showed that the addition of Ferkal in the control treatment led to a significant increasein dry matter production and $\mathrm{P}$ content. However, the best results wereobserved in plants inoculated with AMF and, or Rhizobium, showing significant increases in dry weight and $\mathrm{N}$ and $\mathrm{P}$ content in the shoot, in almost all the inoculated treatments. The indigenous AMF s were more efficient than the G. clarum in promoting plant growth.
\end{abstract}

Index terms: mycorrhizas, degraded areas, phosphorus, nitrogen.

\section{INTRODUÇÃO}

As perturbações naturais que ocorrem em um ambiente são fundamentais para a manutenção, rejuvenescimento e evolução da vida nos ecossistemas (Martinez-Ramos, 1985). Por outrolado, as alterações decorrentes da exploração de recursos minerais demonstram-se mais agressivas, resultando em ambientes com baixa ou nenhuma resiliência, geral mentenecessitando de intervenção antrópica para o restabelecimento da vegetação (Redente et al., 1993). No processo de mineração, denomina-se estéril todo material que não apresenta teor satisfatório do elemento ou compostos em questão (Andrade, 1991).

A lavra ou mineração de argila provoca a degradação física do ambiente, muitas vezes de forma drástica, podendo provocar forte impacto visual, modificações na topografia, erosão do solo, assor eamento de drenagens, dentre outros eventos (Nau \& Sevegnani, 1997; Bal istieri \& Aumond, 1997).

Outra fonte de perturbação ambiental é o descarte inadequado de resíduos industriais. A indústria PURAC- Síntese, localizada às margens do rio Paraíba do Sul, no município de Campos dos Goytacazes (RJ ), que produz ácido láctico a partir de sacarose, gera diariamente grande quantidade de resíduos, para os quais se vem tentando estabelecer uma utilização racional dentro do sistema agropecuário. Dentre os principais resíduos sólidos produzidos, encontram-se o gesso e um resíduo denominado "Ferkal". A similaridadeentreos resíduos desperta o interesse na utilização do Ferkal para fins agrícolas, tendo em vista a comprovada viabilidade da aplicação do gesso no processo produtivo agrícola (Borkert et al., 1987; Braga et al., 1995).

U ma forma de recuperação de áreas degradadas consiste em promover sua revegetação com espécies adequadas a tal propósito. A escol ha dessas espécies é de extrema importância, considerando as características limitantes dos solos degradados. As leguminosas, principalmente as de crescimento rápido, têm demonstrado alta capacidade de adaptação a áreas degradadas por inúmeros processos (Campello, 1999). As espécies da família das leguminosas são reconhecidas como eficientes mel horadoras das características físico-químicas do solo, pois promovem a produção de grande quantidade de biomassa, além de uma vasta exploração do subsol o pel o sistema radicular, acarretando a maior agregação do solo e aumentando a capacidade de retenção deágua (Boni et al., 1994). As leguminosas também podem estabelecer uma dupla simbiose com bactérias diazotróficas, capazes de fixar nitrogênio atmosférico, e com fungos micorrízicos arbusculares (FMAs), sendo estas associações importantes em meios onde o nitrogênio e o fósforo sãolimitantes ao crescimento vegetal (Monteiro, 1990).

As micorrizas podem contribuir para o estabel ecimento, desenvol vimento e crescimento de plantas reintroduzidas por meio do prolongamento 
de suas raízes pelas hifas do fungo micorrízico, que aumentam a área de exploração rizosférica e proporcionam maior absorção de água e nutrientes, permitindo o crescimento vegetal em solos extremamente pobres. Solos que perderam sua camada fértil apresentam reduzi das quantidades de propágulos destes e de outros microrganismos, tornando a introdução de inóculos nestes solos importante do ponto de vista ecológico e de grande interesse tecnológico (Carneiro et al., 1997).

Apesar da importância das associações simbióticas em áreas degradadas em fase de recuperação, ainda são poucos os trabalhos sobre estas associações em espécies utilizadas para revegetar áreas mineradas. Ressalta-se, no entanto, o empenho com que o Centro Nacional de Pesquisa de Agrobiologia - Embrapa Agrobiologia vem enfocando o tema. Notórios trabalhos contribuem para melhoria do "estado da arte" da recuperação deáreas degradadas, seja por meio delevantamentos de leguminosas arbóreas que nodulam e fixam nitrogênio (Franco \& Faria, 1997), seja pela sel eção de FMAs mais efetivos na absorção de nutrientes e água (Franco et al., 1989), seja por trabalhos que visam à seleção de espécies vegetais que promovam o rápido recobrimento do solo e a recuperação das propriedades físicas, químicas e biológi cas das áreas degradadas (Franco et al., 1989; Campello, 1990; Monteiro, 1990; Campello, 1999).

O objetivo do presente trabalho foi avaliar o crescimento de mudas de Mimosa caesal pi niaefol ia (sabiá), inoculadas ou não com FMAs e, ou, rizóbio, crescidas em estéril de extração deargila, adicionado de quatro diferentes doses do resíduo industrial Ferkal.

\section{MATE RIAL E MÉTODOS}

O trabal ho foi realizado em casa de vegetação no "campus" da Universidade Estadual do Norte Fluminense, em Campos dos Goytacazes (RJ ), no período de 14/10/98 a 24/01/99. Foi utilizada a leguminosa arbórea Mimosa caesal piniaefolia (sabiá).

Utilizou-se o delineamento experimental em blocos casualizados, em esquema fatorial $6 \times 4 \mathrm{com}$ três repetições, sendo seis tratamentos microbiológicos (FMAs nativos; FMA Glomus clarum; Rizóbio; FMAs nativos + rizóbio; FMA G. clarum + rizóbio; e controle não inoculado) e quatro doses de Ferkal. A unidade experimental foi composta de duas plantas por vaso.

As mudas foram produzidas em vasos de $6 \mathrm{~L}$, desinfestados com ál cool 70\%. O estéril de extração deargila foi coletado a uma profundi dade de $0-60 \mathrm{~cm}$, de uma área pertencente à cerâmica Caco Manga Ltda., local izada no distrito de U ruraí - Campos dos
Goytacazes (RJ ), cujo sol o é de formação al uvionar.

O resíduo Ferkal foi obtido junto à Industria Purac-Sinteses. Algumas características químicas do resíduo encontram-se no quadro 1.

Tanto o estéril obtido na área de mineração de argila quanto o resíduo F erkal foram postos a secar ao ar e passados em peneiras de 5 e $2 \mathrm{~mm}$, respectivamente. Ao estéril foram adicionadas quatro doses do resíduo Ferkal $\left(0,50,100\right.$ e $200 \mathrm{~g} \mathrm{dm}^{-3}$ de estéril), sendo, a seguir, procedida à análise química do estéril com as diferentes doses de Ferkal adicionadas (Quadro 2).

Foi utilizada uma população nativa de FMAs, isolada de uma área de extração de argila, sendo identificadas as seguintes espécies: Glomus macrocarpum, G. etunicatum e Entrophospora col ombiana. Utilizou-setambém a espécieG. clarum, obtida da coleção da UENF. Os inóculos de FMAs foram previamente multiplicados em vasos de 6,0 L, que continham substrato esterilizado (mistura de sol o e areia lavada na propor ção de 2:1), utilizandosecomo planta hospedeira a Brachiaria decumbens. Como fonte de inóculo para o experimento, foram utilizados $100 \mathrm{ml}$ de uma mistura de solo que continha esporos eraízes col onizadas pelas espécies de FMAs (resultado da multiplicação dos FMAs). O inoculante específico à espécie de leguminosa (BR3405/BR3451) foi obtido junto ao CNPAB EMBRAPA - Seropédica (RJ ).

As sementes de sabiá tiveram sua dormência quebrada por meio de escarificação mecânica por lixamento. Em seguida, foram imersas em hipocl orito

\section{Quadro 1. Características químicas do resíduo} industrial "Ferkal"

\begin{tabular}{lc}
\hline \multicolumn{1}{c}{ Característica } & Valor \\
\hline $\mathrm{pH}\left(\mathrm{H}_{2} \mathrm{O} ; 1: 2,5\right)$ & 8,09 \\
$\mathrm{~N}$ total $\left(\mathrm{g} \mathrm{dm}^{-3}\right)^{(1)}$ & 49 \\
$\mathrm{P}$ total $\left(\mathrm{mg} \mathrm{dm}^{-3}\right)^{(1)}$ & 7.100 \\
$\mathrm{P}$ disponível $\left(\mathrm{mg} \mathrm{dm}^{-3}\right)^{(2)}$ & 8,43 \\
$\mathrm{~K}$ total $\left(\mathrm{mmol}_{\mathrm{c}} \mathrm{dm}^{-3}\right)^{(3)}$ & 6,66 \\
Ca total $\left(\mathrm{mmol}_{\mathrm{c}} \mathrm{dm}^{-3}\right)^{(3)}$ & 8.600 \\
Mg total $\left(\mathrm{mmol}_{\mathrm{c}} \mathrm{dm}^{-3}\right)^{(3)}$ & 350 \\
$\mathrm{~S}$ total $\left(\mathrm{mg} \mathrm{dm}^{-3}\right)^{(3)}$ & $1,51 \times 10^{5}$ \\
$\mathrm{Na}$ total $\left(\mathrm{mmol}_{\mathrm{c}} \mathrm{dm}^{-3}\right)^{(3)}$ & 11,26 \\
Zn total $\left(\mathrm{mg} \mathrm{dm}^{-3}\right)^{(3)}$ & 5,23 \\
Cu total $\left(\mathrm{mg} \mathrm{dm}^{-3}\right)^{(3)}$ & 2,76 \\
Mn total $\left(\mathrm{mg} \mathrm{dm}^{-3}\right)^{(3)}$ & 139 \\
Fe total $\left(\mathrm{mg} \mathrm{dm}^{-3}\right)^{(3)}$ & 1781 \\
CaSO & \\
& 330 \\
\hline (1) Digetal $\left(\mathrm{g} \mathrm{dm}^{-3}\right)^{(3)}$ & \\
perclórica &
\end{tabular}


Quadro 2. Características químicas do estéril de extração de argila nas diferentes doses de Ferkal adicionadas no início (I) e no final (F) do experimento

\begin{tabular}{|c|c|c|c|c|c|c|c|c|}
\hline \multirow{3}{*}{ Característica } & \multicolumn{8}{|c|}{ Dose de Ferkal adicionada ao estéril ( $\mathrm{g} \mathrm{dm}^{-3}$ ) } \\
\hline & \multicolumn{2}{|c|}{$\mathbf{0}$} & \multicolumn{2}{|c|}{50} & \multicolumn{2}{|c|}{100} & \multicolumn{2}{|c|}{200} \\
\hline & $\mathbf{I}$ & $\mathbf{F}$ & $\mathbf{I}$ & $\mathbf{F}$ & $\mathbf{I}$ & $\mathbf{F}$ & $\mathbf{I}$ & $\mathbf{F}$ \\
\hline $\mathrm{pH}$ em $\mathrm{H}_{2} \mathrm{O}$ & 5,27 & 5,42 & 7,26 & 7,60 & 7,60 & 7,81 & 7,80 & 7,91 \\
\hline Matéria orgânica $\left(\mathrm{g} \mathrm{dm}^{-3}\right)$ & 4,33 & n.d. ${ }^{(4)}$ & 5,67 & n.d. & 7,00 & n.d. & 8,33 & n.d. \\
\hline$C\left(g_{d m}^{-3}\right)$ & 2,67 & n.d. & 3,67 & n.d. & 4,00 & n.d. & 4,67 & n.d. \\
\hline $\mathrm{CE}\left(\mathrm{dS} \mathrm{m} \mathrm{m}^{-1}\right)$ & 0,19 & n.d. & 1,77 & n.d. & 1,78 & n.d. & 1,85 & n.d. \\
\hline $\mathrm{H}+\mathrm{Al}\left(\mathrm{mmol}_{\mathrm{c}} \mathrm{dm}^{-3}\right)^{(1)}$ & 29,15 & n.d. & 1,65 & n.d. & 0,00 & n.d. & 0,00 & n.d. \\
\hline$P\left(\mathrm{mg} \mathrm{dm}^{-3}\right)^{(2)}$ & 8,13 & 7,44 & 106,40 & 92,05 & 211,62 & 181,42 & 428,71 & 382,46 \\
\hline $\mathrm{K}\left(\mathrm{mmol}_{\mathrm{c}} \mathrm{dm}^{-3}\right)^{(2)}$ & 42,20 & 44,98 & 49,04 & 55,31 & 49,29 & 59,49 & 55,22 & 52,17 \\
\hline $\mathrm{Ca}\left(\mathrm{mmol}_{\mathrm{c}} \mathrm{dm}^{-3}\right)^{(3)}$ & 14,45 & 11,85 & 354,26 & 249,59 & 589,99 & 466,98 & 865,03 & 853,40 \\
\hline $\mathrm{Mg}\left(\mathrm{mmol}_{\mathrm{c}} \mathrm{dm}^{-3}\right)^{(3)}$ & 19,56 & 10,10 & 21,89 & 13,41 & 22,54 & 12,21 & 24,79 & 20,02 \\
\hline $\mathrm{Na}\left(\mathrm{mmol}_{\mathrm{c}} \mathrm{dm}^{-3}\right)^{(3)}$ & 0,26 & 0,21 & 0,26 & 0,15 & 0,26 & 0,15 & 0,25 & 0,18 \\
\hline $\mathrm{Zn}\left(\mathrm{mg} \mathrm{dm}^{-3}\right)^{(3)}$ & 3,99 & 5,18 & 3,00 & 4,58 & 3,87 & 4,37 & 3,57 & 4,04 \\
\hline $\mathrm{Cu}(\mathrm{mg} \mathrm{dm}-3)^{(3)}$ & 3,07 & 3,33 & 2,94 & 3,21 & 2,95 & 2,98 & 2,33 & 2,38 \\
\hline $\mathrm{Mn}\left(\mathrm{mg} \mathrm{dm}^{-3}\right)^{(3)}$ & 18,0 & 18,37 & 18,35 & 21,48 & 24,32 & 23,84 & 30,82 & 29,55 \\
\hline $\mathrm{Fe}\left(\mathrm{mg} \mathrm{dm}^{-3}\right)^{(3)}$ & 300,0 & 330,48 & 183,59 & 240,86 & 126,99 & 161,51 & 56,71 & 73,18 \\
\hline
\end{tabular}

(1) Acetato de cálcio $1 \mathrm{~N}$ pH 7,0. ${ }^{(2)}$ Extrator Mehlich-1. ${ }^{(3)}$ Extrator KCI 1 N. ${ }^{(4)}$ Não determinado.

de sódio na concentração de 0,5\%, durante 15 min. A inoculação com rizóbio foi feita, misturando-se água destilada aoinoculante, na proporção $1: 1(\mathrm{ml}: \mathrm{g})$, formando uma pasta homogênea. Esta pasta foi misturada às sementes. A semeadura ocorreu em seguida, col ocando-se cerca de 10 sementes em cada vaso, sendo duas por orifício de semeadura. A inoculação com FMAs foi feita durante este processo, adicionando-se a cada orifício aproximadamente $20 \mathrm{ml}$ do inóculo, de acordo com o tratamento microbiológico.

Ao término da segunda semana após a semeadura, foi realizado um desbaste deixando-se apenas duas plântulas (mais vigorosas) por vaso.

A coleta das mudas ocorreu 103 dias após a semeadura. A parteaérea de cada planta foi col ocada individualmente em sacos de papel e, em seguida, foi desidratada em estufa de ventilação forçada, a uma temperatura de $75^{\circ} \mathrm{C}$, por aproximadamente $48 \mathrm{~h}$ (Malavolta et al., 1989). U ma vez desidratado, o material foi pesado, para determinação da matéria seca da parte aérea, e, em segui da, as amostras foram moídas em moinho do tipo Willey, passadas em peneira de 20 "meshes" e armazenadas em frascos hermeticamente fechados. F oram determinados os teores de $\mathrm{N}$ eP da parte aérea. $\mathrm{O} \mathrm{N}$ foi determinado pelo método de Nessler (J ackson, 1958); o P foi determinado col orimetricamente pel o método do mol ibdato, após submeter o material vegetal à oxidação pela digestão sulfúrica (Malavolta et al., 1989).

A pós lavagem em água, subamostras radiculares foram col etadas e conservadas em álcool etílico $50 \%$, para posterior avaliação da col onização mi corrízica pelo método de interseção em placa de Petri reticulada (Giovannetti \& Mosse, 1980).

A colonização radicular com rizóbio foi avaliada por meio da determinação do peso da matéria seca nodular.

O número de microrganismos sol ubilizadores de fosfato foi determinado através da contagem viável em placas de Petri, de acordo com procedimentos adotados por Nahas et al. (1994a).

Para todas as variáveis analisadas, foram ajustadas equações de regressão polinomial, em função das doses de Ferkal adicionadas, adotandose os níveis de significância de $1(* *), 5(*)$ e $10 \%\left({ }^{\wedge}\right)$. Utilizou-se o Sistema de Análise Estatística (SANEST), desenvolvido pelo CIAGRI (Centro de Informática na Agricultura) da Universidade deSão Paulo.

Os dados referentes à percentagem de col onização micorrízica foram previamente submetidos à transformação: $\operatorname{arcsen}(\mathrm{X} / 100)^{0,5}$.

\section{RESULTADOS E DISCUSSÃO}

\section{Colonização micorrízica}

As doses de Ferkal influenciaram a taxa de colonização micorrízica, sendo ajustados modelos 
quadráticos para os tratamentos com dupla inoculação (Figura 1), tendo os seguintes pontos máximos e respectivas doses do resíduo: FMAs nativo + riz. $=77,62 \%$, com 120,34 $\mathrm{g} \mathrm{dm}^{-3}$; FMA G. clarum + rizóbio $=68,36 \%$, com 173,97 g dm ${ }^{-3}$. Para os tratamentos FMAs nativos e FMA G. clarum, ajustaram-se modelos lineares crescentes no intervalo de aplicação de Ferkal utilizado.

A maior taxa de colonização micorrízica nas mudas inoculadas com FMAs nativos frenteao FMA G. clarum pode ser decorrente da maior adaptabilidade do inóculo nativo às condições do substrato, quesão determi nantes no estabel ecimento da simbiose, uma vez que os FMAs são mais específicos para às condições edafocl imáticas do que para o hospedeiro (Le Tacon et al., 1987; Franco \& Faria, 1997).

\section{Nodulação das raízes pelo rizóbio}

As doses de Ferkal influenciaram o peso da matéria seca dos nódulos das mudas de sabiá (Figura 2). Os tratamentos para os quais se ajustaram modelos quadráticos apresentaram o ponto de máximo peso nodular na faixa compreendida entre a aplicação de 115 e $130 \mathrm{~g} \mathrm{dm}^{-3}$ de Ferkal. Tal aspecto pode ser decorrente de alterações de $\mathrm{pH}$ e da disponibilidade de alguns nutrientes. A baixa nodulação em condi ções deacidez pode ser causada tanto pela acidez "per se", como pela deficiência de Ca. Lowther \& Loneragan (1968) demonstraram que a iniciação nodular éaltamente

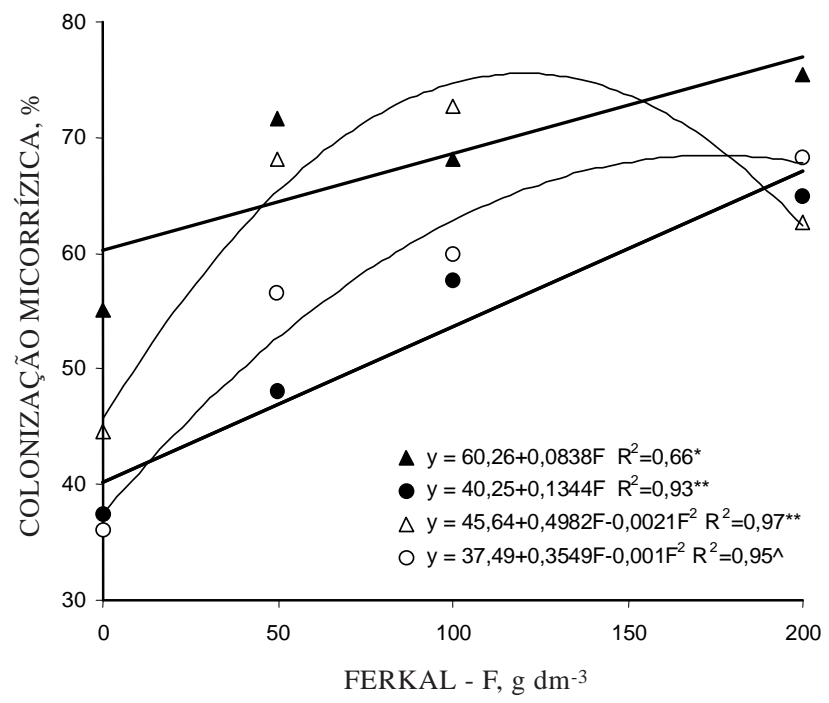

Figura 1. Percentagem do comprimento de raízes de Mimosa caesalpiniaefolia colonizadas por FMAs, nos diferentes tratamentos microbiológicos: FMAs nativos; • FMA G. clarum; $\Delta$ FMAs nativos + Rizóbio; O F MA G. clarum + Rizóbio, considerando as doses de Ferkal. Dados transformados: arc sen $(X / 100)^{0,5}$. Significativos a $1(* *), 5(*)$ e $10 \%\left({ }^{\wedge}\right)$. afetada por baixas concentrações de Ca. A deficiência de Ca também prejudica a adsorsão do rizóbio à superfície radicular, principalmente se combinada a elevadas concentrações de prótons (Siqueira \& Franco, 1988; Marschner, 1995).

Por outro lado, a alcalinidade também exerce efeito detrimental sobre a nodulação. A análise química do substrato empregado revela a diminuição dos níveis de Fe com o aumento nas doses deFerkal. Além deseu papel na fixação biológica de nitrogênio propriamentedita (enzimas do complexo nitrogenase, ferrodoxina, hidrogenase, leg-hemoglobina), a deficiência de Feafeta diretamentea massa nodular, principalmente pela diminuição da divisão das células corticais nos estádios iniciais do desenvolvimento nodular (Tang et al., 1992).

\section{Matéria seca da parte aérea (MSPA)}

A adição de Ferkal promoveu um aumento da MSPA das mudas de sabiá em todos os tratamentos (F igura 2), excetuando-se as mudas-controle, para as quais é apresentada a média de MSPA ao longo das quatro doses do resíduo. Foi ajustado um modelo linear crescente para o aumento de MSPA das mudas inoculadas com FMA G. clarum. Para os demais tratamentos, foram ajustados model os quadráticos com os seguintes pesos máximos e respectivas doses do resíduo: FMAs nativos + rizóbio $=47,97 \mathrm{~g}$, com $187,28 \mathrm{~g} \mathrm{dm}^{-3}$; FMAs nativos $=38,24 \mathrm{~g}$, com $148,60 \mathrm{~g} \mathrm{dm}^{-3}$; FMA G. clarum + rizóbio $=25,62 \mathrm{~g}$, com 146,77 $\mathrm{g} \mathrm{dm}^{-3}$; rizóbio $=13,24 \mathrm{~g}$, com $123,59 \mathrm{~g} \mathrm{dm}^{-3}$.

$\mathrm{Na}$ ausência do resíduo, apesar de não haver influência significativa dos tratamentos microbiológicos, as mudas inoculadas com rizóbio apresentaram mai or produção de MSPA. Tal comportamento pode ser atribuído a um efeito benéfico da fixação bi ológica de nitrogênio, que, segundo Souza \& Silva (1996), é um dos elementos mais limitantes ao desenvolvimento de plantas em áreas degradadas.

O aumento nos teores de $\mathrm{Ca}$, de matéria orgânica e principalmente de $P$, assim como a redução nos teores de Al, possivelmente acarretaram os incrementos na MSPA, apresentados pelas mudas de sabiá, com a adição de Ferkal. A tendência de queda nestes incrementos, demonstrada pelas curvas ajustadas, a partir de determinadas doses do resíduo, em al guns dos tratamentos mi crobiológicos, pode ser atribuída à contínua el evação do pH e às conseqüentes alterações na disponibilidade de nutrientes em condi ções de alcal inidade (Marschner, 1995). Santos et al. (1998), analisando um experimento com mudas de leguminosas, também concluíram que aumentos progressivos nos teores de $\mathrm{P}$ do substrato podem deprimir os benefícios da dupla inoculação, rizóbio x micorriza, ao hospedeiro.

A maior exploração do meio edáfico, promovida pela micorrização, torna a planta hospedeira mais hábil na competição por recursos, bem como mais 

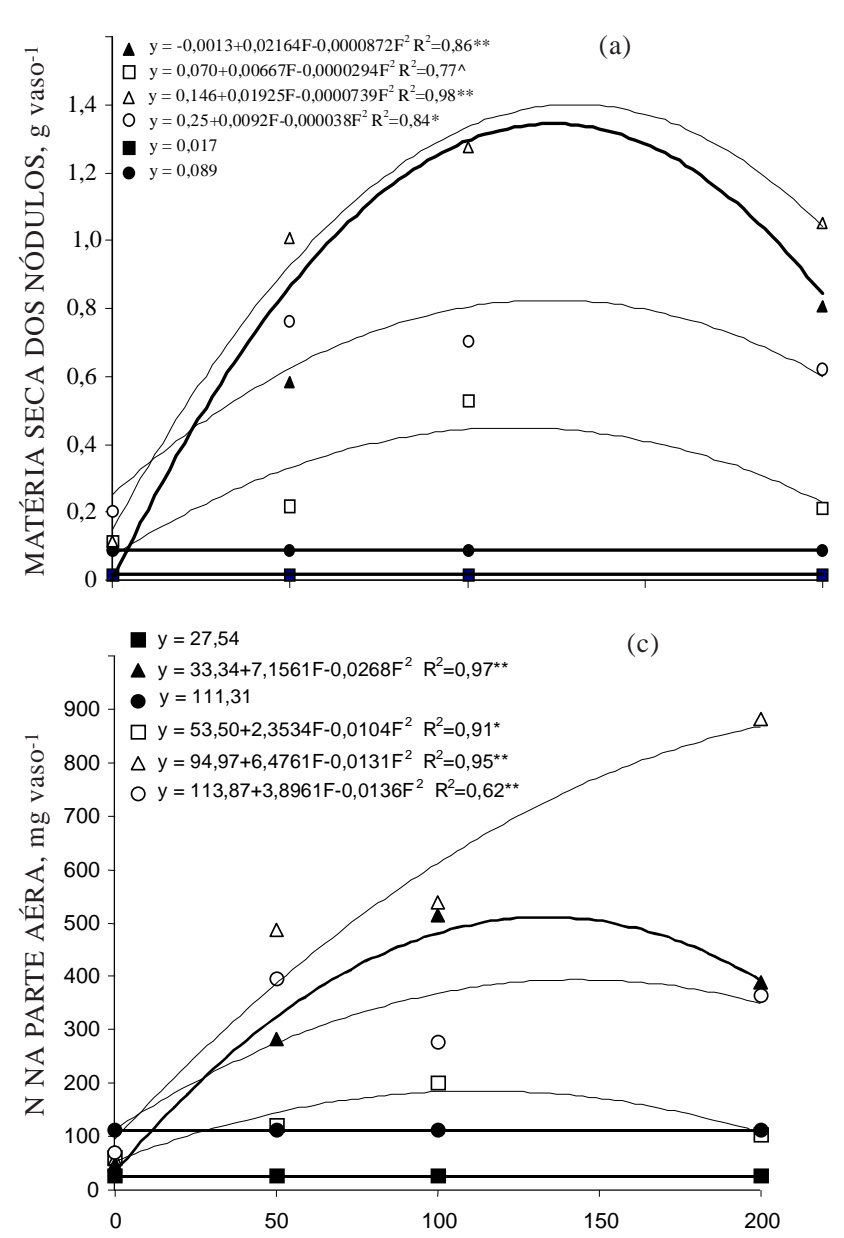

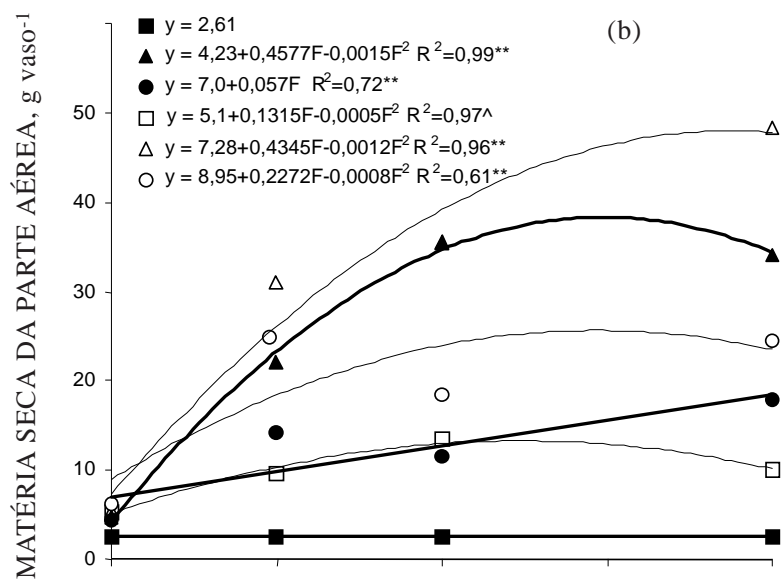

(d)

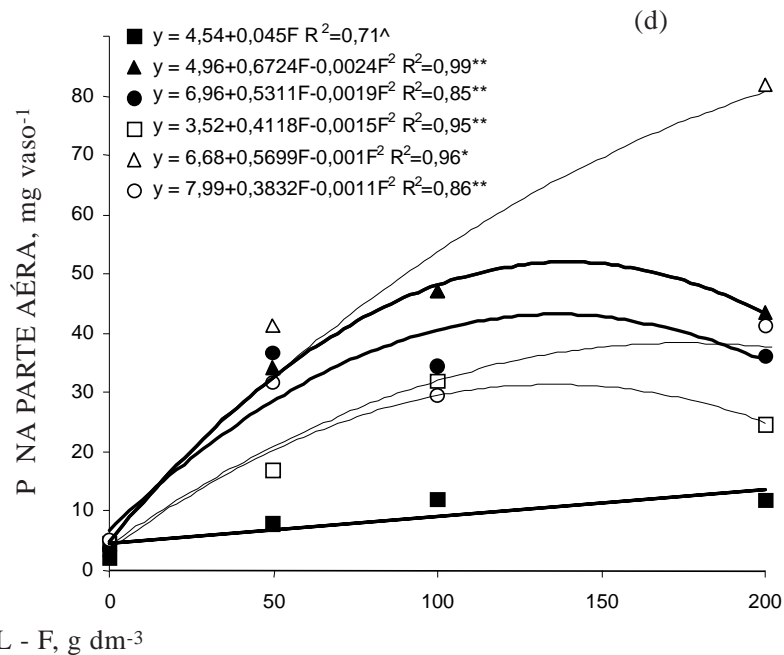

Figura 2. Peso da matéria seca de nódulos radiculares (a), peso da matéria seca da parte aérea (b), teores de nitrogênio (c) e fósforo (d) na parte aérea de Mimosa caesal pi niaefolia, nos di ferentes tratamentos microbiológicos: Controle; • FMA G. clarum; FMAs nativos; Rizóbio; O F MA G. clarum + Rizóbio; e, $\Delta$ F MAs nativos + Rizóbio, considerando as doses de Ferkal, adicionadas a estéril de extração de argila. Significativos a $1(* *), 5(*)$ e $10 \%\left({ }^{\wedge}\right)$.

tol erantea condições adversas (Souza \& Silva, 1996), sendo, provavelmente, esta a razão pela qual as mudas micorrizadas revelaram valores de MSPA superiores aos das mudas-controle.

A nodulação apresentada pelas mudas inoculadas com FMAs nativos e com FMA G. clarum justifica a equivalência destas mudas na produção de MSPA com as inoculadas com $F$ M As nativos + rizóbio e com FMA G. clarum + rizóbio, respectivamente. No presente estudo, o tratamento com inoculação de FMAs nativos + rizóbio selecionado mostrou estimativa para MSPA máxima, superior à da inoculação só com FMAs nativos, quando empregadas maiores doses de Ferkal.

\section{Teores de $\mathbf{N}$ e $\mathbf{P}$ na parte aérea}

Os teores de $\mathrm{N}$ e $\mathrm{P}$ na parte aérea das mudas de sabiá foram, de maneira geral, influenciados tanto pelos tratamentos microbiológicos quanto pela adição de Ferkal (Figura 2). A adição do resíduo proporcionou um aumento gradual na concentração de $\mathrm{P}$ e aumentou os teores de carbono e de matéria orgânica do substrato (Quadro 2). O aumento expressivo na disponibilidade deP do substrato, em função do aumento das doses de Ferkal, pode estar relacionado com o aumento do número de microrganismos solubilizadores de fosfato do solo (Figura 3). Tal aumento pode ser devido ao aumento de $\mathrm{pH}$ do solo. Nahas et al. (1994b) demonstraram que o número de microrganismos produtores de fosfatases aumentou com a el evação do pH do solo.

A aplicação de Ferkal reduziu também a possibilidade de efeitos detrimentais da presença de Al, além de elevar a disponibilidade de Ca. Todos estes fatores, isolados ou em conjunto, podem ter contribuído para os maiores teores dos elementos analisados na parte aérea das mudas de sabiá. 


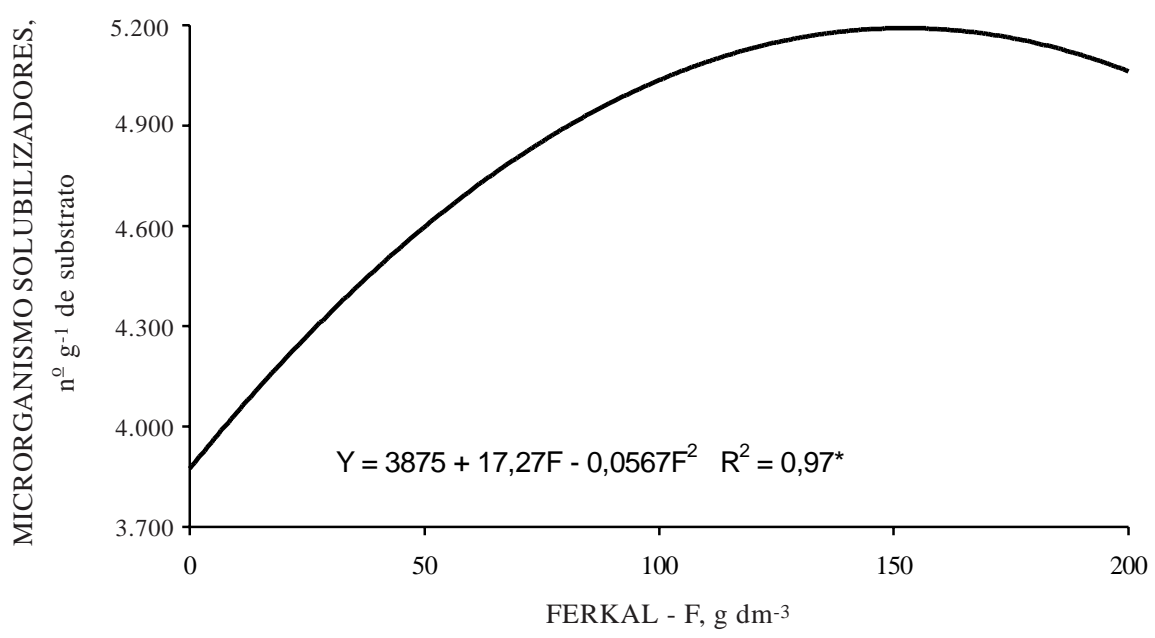

Figura 3. Número de microrganismos solubilizadores de fosfato no substrato, considerando as doses de Ferkal. Significativo a $5 \%(*)$.

A tendência dereduçãonos teores deN eP, a partir da adição de determinadas doses do resíduo, pode ser resultado, principalmente, do excesso de Ca e do $\mathrm{pH}$ elevado sobre a absorção de nutrientes. O desenvolvimento radicular também pode ter sido afetado não só pela alta concentração deCa e el evado pH, mas também pela deficiência de outros el ementos, como o $\mathrm{Fe}$, comprometendo o desenvolvimento das mudas. A deficiência de Fe é um problema comum em solos calcários (Marschner, 1995) e suas conseqüências sobre o sistema radicular são percebi das pela ini bição do al ongamento das raízes e pelo aumento do diâmetro dos ápices radiculares (Römreld \& Marschner, 1981; Chaney et al., 1992), comprometendo a absorção de nutrientes.

Com a adição de F erkal, houve uma el evação de teor de $\mathrm{P}$ na parte aérea das mudas, decorrente, possivel mente, da el evação gradual dos teores desse elemento no substrato.

As mudas inoculadas com rizóbio apresentaram aumentos nos teores de N eP com a adição deF erkal ao substrato(Figura 2), demonstrando ofavorecimento ao desenvolvimento do par simbionte com as alterações promovidas no substrato, pela adição do resíduo. A melhoria das condições nutricionais do substrato com a adição do resíduo pode ter contribuído para a elevação do teor dos elementos analisados, principalmente do P. Esta melhoria no "status" nutricional da planta hospedeira pode ter favorecido a eficiência da fixação biológica de nitrogênio (FBN), acarretando contribuições mais efetivas desse processo no acúmulo de $\mathrm{N}$ na planta. A diminuição do teor de Al do substrato reduz os riscos de efeitos tóxicos desse el emento sobre as bactérias diazotróficas (geralmente sensíveis). 0 aumento da disponibilidade de $\mathrm{P}$, em virtude do al to requerimento energético da FBN e de Ca (críticos nos processos de infecção e formação de nódulos) pode resultar na melhor eficiência da simbiose rizóbio-leguminosa.

As mudas inoculadas com FM As nativos + rizóbio e com F MAs nativos foram as que apresentaram os maiores teores de $\mathrm{N}$ eP na parte aérea, com a adição do resíduo. Muitos mecanismos podem contribuir para a maior capacidade de absorção das plantas col onizadas por FMAs, destacando-se o aumento da superfície de absorção e exploração do solo, o armazenamento temporário de nutrientes na biomassa fúngica, evitando imobilização (química/ biológica) ou a lixiviação. Destacam-se, também, o aumento da capacidade de absorção da raiz, pela redução do Km (aumento no influxo); o aumento na longevidade das raízes (Siqueira \& Franco, 1988) e as mudanças morfológicas no sistema radicular, aumentando o número de raízes terciárias e quaternárias e elevando, conseqüentemente, sua efetividade na exploração do sol o (Berta et al., 1995). Os FMAs também favorecem indiretamente a absorção de nutrientes por meio da amenização de eventuais efeitos adversos do pH, da concentração deAl e Mn, da salinidade e do estresse hídrico sobre o crescimento da planta hospedeira.

A maior capacidade de absorção de elementos pouco móveis no solo, principalmente o $P$, é o benefício nutricional mais conhecido da col onização por FMAs (Harley \& Smith, 1983). Além do maior volume de solo explorado pelas hifas, Bolan et al. (1984) sugeriram que o micélio externo dos FMAs possa absorver fosfato em baixas concentrações mais efetivamente do que as raízes. A efetividade dos FMAs em fornecer $\mathrm{P}$ para a planta hospedeira depende muito da espécie e pode ser atribuída não só à taxa de colonização, mas também ao desenvolvimento e à atividade das hifas externas, 
às taxas de transporte nas hifas e às taxas de intercâmbio de sol utos na interface arbúsculo-célula radicular hospedeira.

Os maiores teores de $\mathrm{P}$ e, principalmente, de $\mathrm{N}$, apresentados pelas mudas inoculadas com FMAs nativos + rizóbio, são, provavelmente, conseqüência de um efeito sinergístico entre os simbiontes, tendo a melhoria no "status" nutricional da planta hospedeira favorecido osuprimento de fotoassimilados aos microrganismos simbiontes, e vice-versa. Costa \& Paulino (1992) comprovaram que a dupla inoculação aumentou significativamente os teores de N e P na parte aérea de Leucaena leucocephala.

As mudas submetidas a uma dupla inoculação com FMA G. clarum + rizóbio revelaram teores de $\mathrm{N}$ superiores aos apresentados pelas mudas inoculadas apenas com F MA G. clarum, sugerindo, novamente, a possível contribuição da FBN no suprimento desse el emento às mudas de sabiá.

\section{CONCLUSÕES}

1. A adição das doses de Ferkal sem a inoculação das mudas com FMAs e, ou, rizóbio (tratamento controle) aumentou, significativamente, o teor de $P$ na parte aérea. Entretanto, os benefícios foram maiores nas mudas inoculadas com os microssimbiontes, as quais apresentaram aumentos significativos no peso da matéria seca e nos teores de $\mathrm{N}$ e $\mathrm{P}$ em relação ao tratamento-controle.

2. Os F MAs nativos foram mais eficientes do que o FMA G. clarum, sendo a dupla inoculação, FMAs nativos + rizóbio, o tratamento que promoveu o melhor crescimento de M. caesal pi niaefolia.

\section{AGRADE CIMENTOS}

À F undação de Amparoà Pesquisa do Estado do Rio de J aneiro (FAPERJ ), pelo auxílio financeiro; à EMBRAPA Agrobiologia, pelo fornecimento dos inócul os de rizóbio, eà Dra. Rosilaine Carrenho, pela identificação das espécies nativas de FMAs.

\section{LITERATURA CITADA}

ANDRADE, L.A.B.L. Influência da associação micorrízica e da adição de matéria orgânica sobre o crescimento de Brachiaria decumbens em estéril de mineração de ferro e bauxita. Viçosa, U niversidade F ederal de Viçosa, 1991. 53p. (Tese de Mestrado)
BALISTIERI, P.R.M.N. \& AUMOND, J J . Recuperaçãoambiental em mina de argila, Doutor Pedrinho - SC. In: SIMPÓSIO NACIONAL DE RECUPERAÇÃO DE ÁREAS DEGRADADAS, 3., Ouro Preto, 1997. Anais. Viçosa, Fol ha Florestal, p.42-51.

BERTA, G.; TROTTA, A.; FUSCONI, A.; HOOKER, J .E.; MUNRO, M.; ATKINSON, D.; GIOVANETTI, M.; MORINI, S.; FORTUNA, P. \& TISSERANT, B. Arbuscular mycorrhizal induced changes to plant growth and root system morphology in Prunus cerasifera. TreePhysiol., 15:281-293, 1995.

BOLAN, N.S.; ROBSON, A.D.; BARROW, N.J . \& AYLMORE, L.A.G. Specific activity of phosphorus in mycorrhizal and non-mycorrhizal plants in relation to the availability of phosphorus to plants. Soil Biol. Biochem., 16:299-304, 1984.

BONI, N.R.; ESPÍNDOLA, R. \& GUIMARÃES, E.C. Uso de leguminosas na recuperação de um solo decapitado. In: SIMPÓSIO SUL-AMERICANO, 1., SIMPÓSIO NACIONAL DE RECUPERAÇÃO DE ÁREAS DEGRADADAS, 2., Foz do I guaçu, 1994. Anais. Curitiba, FUPEF, 1994. p.563-568.

BORKERT, C.M.; PAVAN, M.A. \& LANTMANN, A.F. Considerações sobre o uso de gesso na agricultura. Piracicaba, Associação Brasileira para a Pesquisa da Potassa e do Fosfato - POTAFÓS, 1987. (Informações agronômicas, 40)

BRAGA, F.A.; VALE, F.R. \& MUNIZ, J .A. Movimentação de nutrientes no solo, crescimento e nutrição mineral do eucal ipto, em função de doses de gesso e níveis de irrigação. R. Bras. Ci. Solo, 19:69-78, 1995.

CAMPELLO, E.F.C. Potencial de utilização de espécies actinorrízicas em sol os degradados. Curitiba, U niversidade Federal do Paraná, 1990. 83p. (Tese de Mestrado)

CAMPELLO, E.F.C. A influência de leguminosas arbóreas fixadoras de nitrogênio na sucessão vegetal em áreas degradadas na Amazônia. Viçosa, U niversidadeF ederal de Viçosa, 1999. 121p. (Tese de Doutorado)

CARNEIRO, M.A.C.; SIQUEIRA, J .O.; CURI, N. \& MOREIRA, F.M.S. Fungos micorrízicos e superfosfato no acúmulo de nitrogênio e no crescimento de plantas herbáceas em solo degradado. In: SIMPÓSIO NACIONAL DE RECUPERAÇÃO DE ÁREAS DEGRADADAS, 3., Ouro Preto, 1997. Anais. Viçosa, Fol ha Florestal, p.231-239.

CHANEY,R.L.; CHEN, Y.; GREEN, C.E.; HOLDEN, M.J .; BELL, P.F.; LUSTER, D.G. \& ANGLE, J .S. Root hairs on chlorotic tomatoes are an effect of chlorosis rather than part of the adaptive Fe-stress response. J. Plant. Nutr., 15:1857-1875, 1992.

COSTA, N.L. \& PAULINO, V.T. Response of leucaena to single and combined inoculation with rhizobium and mycorrhiza. Nitrogen Fixing Tree Res. Rep., 10:45-46, 1992.

FRANCO, A.A.; CAMPELLO, E.F.C.; MONTEIRO, E.M.S.; CUNHA, C.O., CAMPOS NETO, D. \& DÖBEREINER, J . Nodulated legumetrees for the recuperation of acid tropical soils. In: THE NORTH AMERICAN SYMBIOTIC NITROGEN FIXATION CONFERENCE, 12., 1989, IOWA. Proceedings. IOWA: I owa State University, 1989. p.70. 
FRANCO, A.A. \& FARIA, S.M. The contribution of $\mathrm{N}_{2}$-fixing tree legumes to land reclamation and sustainability in the tropics. Soil Biol. Biochem., 29:897-903, 1997.

GIOVANNETTI, M. \& MOSSE, B. An evalution of techniques for measuring $V A$ mycorrhizal infection in roots. New Phytol., 84:489-500, 1980.

HARLEY, J .L. \& SMITH, S.E. Mycorrhizal symbiosis. New York, Academic Press, 1983. 483p.

J ACKSON, M.L. Soil chemical analysis. New J ersey: Prentice Hall, 1958. 489p.

LeTACON, F.; GARBAYE, J . \& CARR, G. The use of mycorrhizas in the temperate and tropical forest. Symbiosis, 3:19792006, 1987.

LOWTHER, W.L. \& LONERAGAN, J .F. Calcium and nodulation in subterranean clover (Trifolium subterraneum L.). Plant Physiol., 43:1362-1366, 1968.

MALAVOLTA, E.; VITTI, G.G. \& OLIVEIRA, S.A. Avaliação do estado nutricional das plantas: princípios e aplicações. Piracicaba, Associação Brasileira para Pesquisa da Potassa e do Fósforo, 1989. 201p.

MARSCHNER, H. Mineral nutrition of higher plants. 2.ed. London, Academic Press, 1995. 889p.

MARTINEZ-RAMOS, M.C. Claros, ciclos vitales de los árboles tropicales y regeneración natural de las selvas altas perenifolias. In: GOMEZ-POMPA, A. \& DEL AMO, S., eds. Investigaciones sobre la regeneración de selvas en Vera Cruz. Mexico, Alhambra Mexicana, 1985. p.116-143.

MONTEIRO, E.M.S. Resposta de leguminosas arbóreas à inoculação com rizóbio e fungos micorrízicos em solo ácido. I taguaí, Universidade Federal Rural do Rio deJ aneiro, 1990. 221p. (Tese de Doutorado)

NAHAS, E.; CENTURION, J .F. \& ASSIS, L.C. Microrganismos solubilizadores de fosfato e produtores de fosfatases de vários solos. R. Bras. Ci. Solo, 18:43-48, 1994a.
NAHAS, E.; CENTURION, J.F. \& ASSIS, L.C. Efeito das características químicas dos sol os sobre os microrganismos solubilizadores de fosfato e produtores de fosfatases. R. Bras. Ci. Solo, 18:49-53, 1994b.

NAU, S.R. \& SEVEGNANI , L. Vegetação recol onizadora em mina de argila e propostas para recuperação ambiental. In: SIMPÓSIO NACIONAL DE RECUPERAÇÃO DE ÁREAS DEGRADADAS, 3.,Ouro Preto, 1997. Viçosa, Fol ha Florestal, 1997. p.54-66.

REDENTE, E.F.; MCLENDON, T. \& DePUIT, E.J . Manipulation of vegetation community dynamics for degraded land rehabilitation. In: SIMPÓSIO BRASILEIRO DE PESQUISA FLORESTAL, 1., Belo Horizonte, 1993. Anais. Viçosa, Sociedade de I nvestigações Florestais, 1993. p.265-278.

RÖMHELD, V. \& MARSCHNER, H. Rhythmic iron stress reaction in sunflower at suboptimal iron supply. Physiol. Plant., 53:347-353, 1981.

SANTOS, D.R.; MOREIRA, F.M.S. \& SIQUEIRA, J .O. Fósforo, fungo micorrízico e rizóbio no crescimento, nodulação e fixação biológica do nitrogênio em Sesbania virgata (Cav.) e Sesbania rostrata (Bram.). In: REUNIÃO BRASILEIRA DE FERTILIDADE DO SOLO E NUTRIÇÃO DE PLANTAS, 23., REUNIÃO BRASILEIRA SOBRE MICORRIZAS, 7., SIMPÓSIO BRASILEIRO DE MICROBIOLOGIA DO SOLO, 5., REUNIÃO BRASILEIRA DE BIOLOGIA DO SOLO, 2., Caxambú, 1998. Anais. Lavras, UFLA/SBCS/SBM, 1998. p.772-772.

SIQUEIRA, J.O. \& FRANCO, A.A. Biotecnologia do solo: fundamentos e perspectivas. Brasília, Ministério da Educação e Cultura, 1988. 235p.

SOUZA, F.A. \& SILVA, E.M.R. Micorrizas arbusculares na revegetação de áreas degradadas. In: SIQUEIRA, J .O., ed. Avanços em fundamentos eaplicação de micorrizas. Lavras, UFLA / DCS, 1996. p.255-290.

TANG, C.; ROBSON, A.D.; DILWORTH, M.J . \& KUO, J . Microscopic evidence on how iron deficiency limits nodule initiation in Lupinus angustifolius L. New Phytol ., 121:457467, 1992. 
A. Z. PRALON \& M. A. MARTINS

R. Bras. Ci. Solo, 25:55-63, 2001 